\title{
A Double FFT Procedure to Improve the Sensitivity of a Tunable Diode Laser Open Path Spectrometer for Sensing Local Atmospheric Gas Concentrations on Mars
}

\author{
Youhua Chen ${ }^{1}$, Samira Mahdi ${ }^{2}$, Gary Anderson ${ }^{3}$ \\ 1 - Graduate Institute of Technology, University of Arkansas at Little Rock, AR 72204,yxchen1@ualr.edu \\ 2 - Department of Applied Science, University of Arkansas at Little Rock, AR 72204, samahdi@ualr.edu \\ 3 -Department of System Engineering, University of Arkansas at Little Rock, AR 72204, gtanderson@ualr.edu
}

\begin{abstract}
A double FFT procedure is developed to improve the sensitivity of a spectrometer to measure atmospheric gases. Simulations show the DFFT reduces the effects of $1 / \mathrm{f}$ noise and increase the sensitivity of the measurement.

OCIS codes: (280.1100) Aerosol detection; (300.6260) Spectroscopy, diode lasers.
\end{abstract}

\section{Introduction}

An in-situ measurement system was previously proposed to measure gas concentrations in the Martian atmosphere that could be biogenic gases [1]. The heart of the system is a Tunable Diode Laser Spectrometer (TDLS), which works by modulating the wavelength of light emitted by a near-infrared diode laser. The laser is pointed at a retroreflector, which reflects light back to a photodiode placed next to the laser. As the wavelength of emitted light passes through the absorption peak of a gas of interest, the intensity of the light received by the photodiode will decrease if any gas is present. Because the measurement needs to detect a small dip on a large background signal, signal processing techniques are usually used to detect the gas signal in the frequency domain.

For our project, the TDLS is placed on a mobile robot, which is moved around the local terrain to make gas readings through the Martian atmosphere at measurement distance as small as 20 meters to as long as $1 \mathrm{~km}$. Because the system is to be used for space applications, it has requirements that most open path spectrometers do not: it must be robust, simple to operate, have low power requirements, be small in size and have low mass. These constrains preclude the use of many high-resolution spectrometer techniques such as using reference gas cells and high frequency operation.

Noise is a major factor limiting measurement sensitivity in TDLS systems [2]. Due to their quantum properties, diode laser exhibit a large $1 /$ f noise spectrum. Many systems try to minimize this effect by using high frequency techniques, but the requirements of our system make this difficult. We therefore are examining techniques to reduce the effects of $1 / \mathrm{f}$ noise on measurements made at lower frequencies, e.g., $500 \mathrm{~Hz}$.

This paper describes a new signal processing method to take Double Fast Fourier Transform (DFFT) on the raw sampling data of the open path spectrometer. The advantage of the method is that it transforms the $1 / \mathrm{f}$ spectrum of the laser noise to a nearly flat spectrum. One emphasis in this work is to improve the sensitivity of measurement harmonics amplitude to gas concentration. The next section describes the open path spectrometer. Section 3 proposes the DFFT procedure, while section 4 shows computer simulations.

\section{Setup of the TDLS}

The TDLS consists of an analog input/output device (NI DAQ USB 6211), a DFB near-infrared diode laser (NLK1655STG with nominal wavelength $1532.68 \mathrm{~nm}$ for ammonia), a laser driver (Wavelength Electronics LDTC1020), a photo diode (Thorlabs SM05PD5A), a customized retroreflector with multiple small corner cube prisms and an electronic PCB board that performs amplification, analog filtering and level shifting functions. The setup of the system is shown in Fig. 1. The DAQ device has 16-bit analog input and output with $250 \mathrm{kS} / \mathrm{s}$ maximum sampling rate. The DFB diode laser is driven by a sinusoidal modulation of frequency $f_{\text {mod }}=500 \mathrm{~Hz}$ to sweep the optical frequency across the desired gas absorption line. The wavelength coefficient of the tunable diode laser is $0.01 \mathrm{~nm} / \mathrm{mA}$. The total injection current $i_{L D}$ is given by

$$
i_{L D}(t)=\alpha\left[V_{\text {off }}+V_{\text {amp }} \sin \left(2 \pi f_{\text {mod }} t\right)\right]
$$

where $\alpha$ is the transfer factor of the laser driver from setting voltage to injection current, $V_{\text {off }}$ is the voltage offset in $\mathrm{mV}$, and $V_{a m p}$ is the voltage amplitude of the sinusoidal modulation in $\mathrm{mV}$. Because each period of the sine wave is assumed to have 64 data points, the sampling rate $f_{s}$ has $f_{s}=64 f_{\text {mod }}$. 


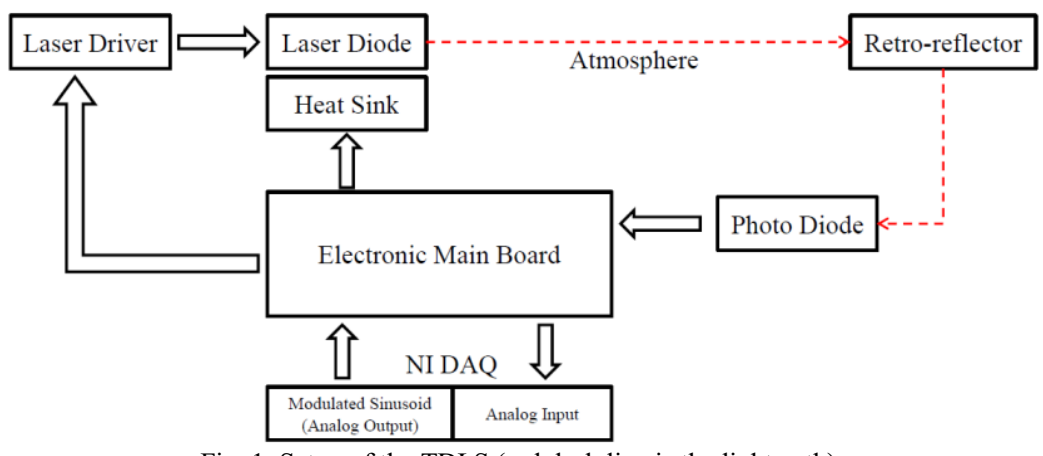

Fig. 1. Setup of the TDLS (red dash line is the light path).

\section{Description of the Double FFT Procedure}

The DFFT procedure begins by applying $500 \mathrm{~Hz}$ sine wave to the laser diode, driving the emitted light through the absorption peak of a gas of interest. A Fast Fourier Transform (FFT) is then applied to the intensity data from the photodiode. The amplitudes of the first several harmonics (4, 8 or 16) are then selected and a second FFT is applied to this data, which converts the $1 / \mathrm{f}$ noise to a flat or low amplitude periodic spectrum. In the mean while, the profile of the $1 / \mathrm{f}$ noise is converted to flat. After that, the characteristic features are extracted such as the sum of harmonics, the mean value of harmonics and the maximum value of harmonics. A linear relationship between the sum of harmonics and gas concentrations was empirically determined, with the gas concentration $g$ is calculated by $g=f^{-1}(h)$. A typical linear measurement function $f(h)$ is $h=A g+B$, where $A$ is the slope of the linear fitting curve.

\section{Computer Simulations}

To evaluate the effective sensitivity of the DFFT procedure, computer simulations are made based on the Beer Lambert Law [3] as

$$
I=I_{0} e^{-\beta \sigma N L},
$$

where $I_{0}$ and $I$ (unit: $\mathrm{mW} / \mathrm{mm}^{2}$ ) are the intensity of incident light and transmitted light in time domain, respectively; $\beta$ (unit: $\mathrm{cm}^{-3} / \mathrm{ppm}$ ) is the factor to convert the unit from ppm to $\mathrm{cm}^{-3} ; \sigma$ (unit: $\mathrm{cm}^{2}$ ) is the absorption cross section (assumed to be Gaussian distributed on light wavelength to match Earth-based test measurements); $N$ (unit: parts per million) is the gas concentration, and $L$ (unit: $\mathrm{cm}$ ) is the light path length. Computer simulations were run to determine the sum of harmonics for fixed gas concentrations. Repeating the simulations with various gas concentrations, multiple data points are computed and their fitting curves are derived to describe the relation of gas concentration and the sum of harmonics (Fig. 3 and Fig. 4). Since the wavelength range of the diode laser used in the TDLS covers the absorption lines of ammonia, all of the following simulations are aimed to detect the concentration of ammonia.

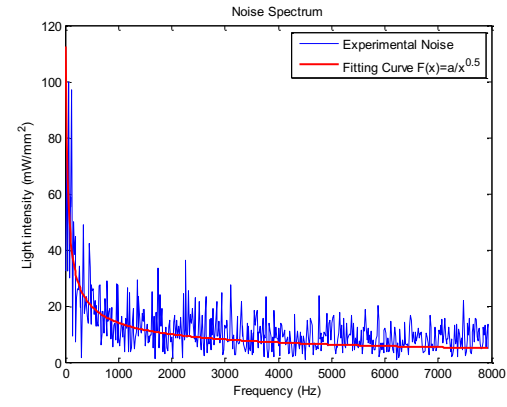

a. Experimental noise spectrum. The function of the fitting curve is $443.3 / x^{0.5}$.

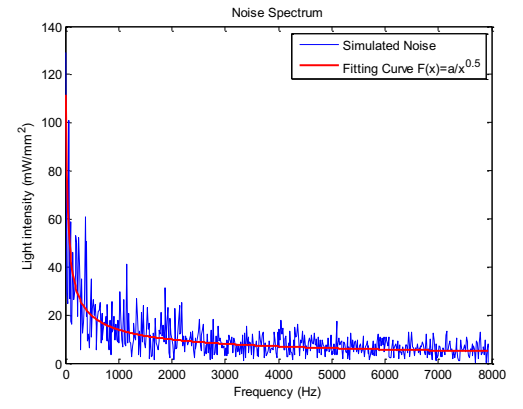

b. Simulated noise spectrum. The function of the fitting curve is $439.5 / x^{0.5}$.

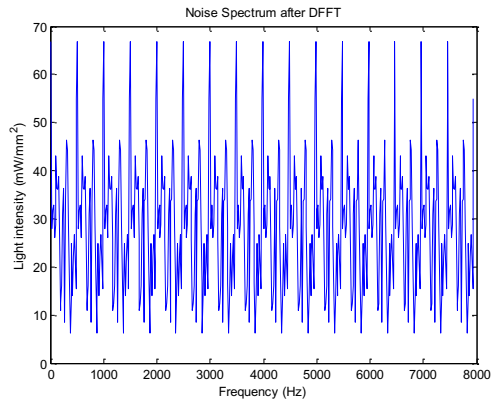

c. Noise spectrum after DFFT with 16 harmonics selected.

Fig. 2. 1/f noise spectrum of the light intensity.

$1 / \mathrm{f}$ noise was added to the simulated data points, with the $1 / \mathrm{f}$ distribution function coming either from experimental noise data or from simulated noise with similar amplitudes. The spectrum of the noise always follows a 1/f profile (Fig. 2a and Fig. 2b). The 1/f noise is transformed to a flat or small-amplitude periodic profile by the 
DFFT (Fig. 2c). Thus the signals are better evaluated at lower frequencies in the DFFT domain because of smaller noise signal.

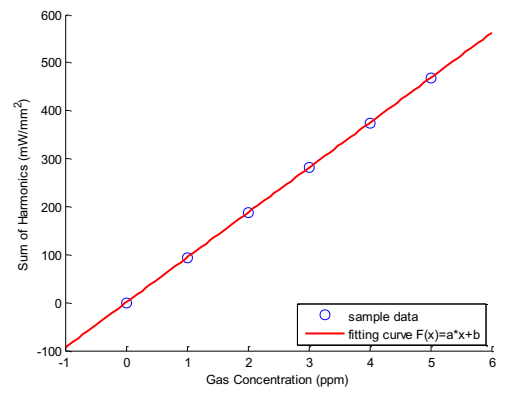

a. DFFT without simulated noise and 16 harmonics selected. The function of the fitting curve is $93.56 x-0.1301$.

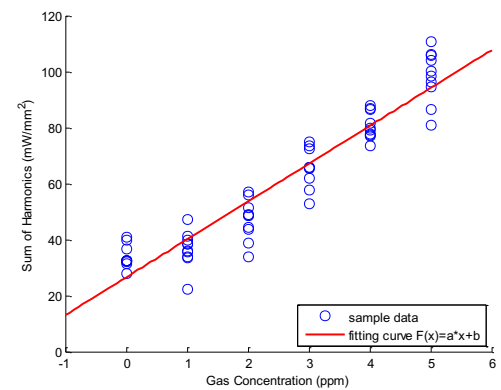

b. Single FFT with simulated noise and 16 harmonics selected. The function of the fitting curve is $13.51 x+26.584$.

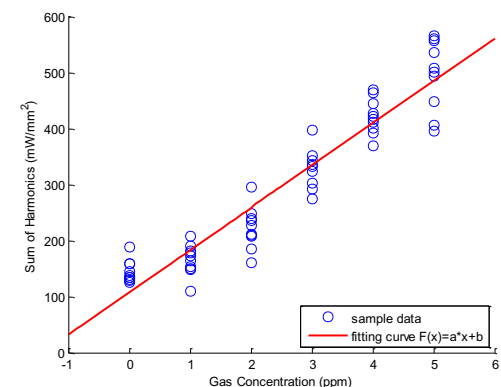

c. DFFT with simulated noise and 16 harmonics selected. The function of the fitting curve is $75.35 x+108.1$.

Fig. 3. Simulation results of gas concentration vs. sum of harmonics.

Fig. 3 shows simulation results using single FFT and DFFT analysis with and without 1/f noise present. All data points are plotted for ammonia gas concentration vs. the sum of harmonics. For each given gas concentration, the simulation is repeated 10 times, so that 10 points are generated using different noise signals. If there is no noise, a perfect linear fitting curve is obtained (Fig. 3a) with an average estimation error $9.265 \times 10^{-4}$ ppm. The linear function $h=A g+B$ is derived for different simulation conditions (i.e., a single FFT or DFFT, using 4, 8 or 16 harmonics). The estimation error is the difference between actual gas concentration and the gas concentration of the point on the linear fitting curve (measured gas concentration) for the same sum of harmonics. The slope $A$ is a critical parameter to indicate the measurement sensitivity. The measure sensitivity is proportional to the slope of the fitting curve.

Tab. 1 shows the slope and the average estimation error, where the maximum slope and the minimal estimation error are obtained by using DFFT and calculating the sum of 16 harmonics. It matches the assumption that most signal information of the absorption peak is presented on harmonics.

Tab. 1. Sensitivity factor and estimation error

\begin{tabular}{|l|c|c|}
\hline & Slope,$\left(\mathrm{mW} / \mathrm{mm}^{2}\right) / \mathrm{ppm}$ & Average estimation Error, $\mathrm{ppm}$ \\
\hline Single FFT, 4 Harmonics & 10.24 & 0.4663 \\
\hline Single FFT, 8 Harmonics & 12.98 & 0.4576 \\
\hline Single FFT, 16 Harmonics & 13.51 & 0.4524 \\
\hline DFFT, 4 Harmonics & 21.96 & 0.4272 \\
\hline DFFT, 8 Harmonics & 40.92 & 0.4207 \\
\hline DFFT, 16 Harmonics & $\mathbf{7 5 . 3 5}$ & $\mathbf{0 . 4 0 8 1}$ \\
\hline
\end{tabular}

\section{Conclusion}

The new DFFT procedure is effective to reduce the $1 / \mathrm{f}$ noise and improve the measurement sensitivity of the TDLS to detect biogenic gases like ammonia. The sum of harmonics is a critical feature to present the gas concentration. By simulating light absorption intensity, linear measurement functions from gas concentration to the sum of harmonics are derived to be used for real-time measurement. Experiments with different ammonia concentration will be made to find actual measurement functions.

\section{Acknowledgements}

This work was supported by the research project - Mobile Surveying for Atmospheric and Surface Gases of Biological Origins founded by NASA (NASA Cooperative Agreement NNX09A072A).

\section{References}

[1] G. Anderson, et al., "A mobile robot system for remote measurements of ammonia vapor in the atmosphere" in Systems, Man and Cybernetics, 2006. SMC '06. IEEE International Conference on. 2006.

[2] P. Werle, et al., "Signal processing and calibration procedures for in-situ diode-laser absorption spectroscopy," Spectrochimica Acta A 60, 1685-1705 (2004).

[3] J. T. Houghton, The physics of atmospheres (2nd ed., Cambridge University Press, 1986), Cambridge, page 271. 